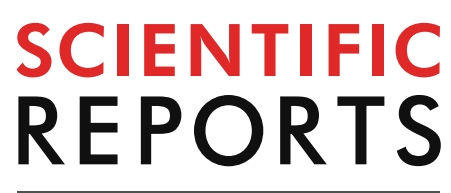

natureresearch

Check for updates

\title{
Growth retardation-responsive analysis of mRNAs and long noncoding RNAs in the liver tissue of Leiqiong cattle
}

\author{
Lingxuan Kong ${ }^{1,2,3}$, Guangbin Liu ${ }^{1,2,3}$, Ming Deng ${ }^{1,2}$, Zhiquan Lian ${ }^{1,2}$, Yinru Han ${ }^{1,2}$, \\ Baoli Sun ${ }^{1,2}$, Yongqing Guo ${ }^{1,2}$, Dewu Liu ${ }^{1,2 \bowtie}$ \& Yaokun $\mathrm{Li}^{1,2 \bowtie}$
}

As an important type of non-coding RNA molecule, long non-coding RNAs (IncRNAs) have varied roles in many biological processes, and have been studied extensively over the past few years. However, little is known about IncRNA-mediated regulation during cattle growth and development. Therefore, in the present study, RNA sequencing was used to determine the expression level of mRNAs and IncRNAs in the liver of adult Leiqiong cattle under the condition of growth retardation and normal growth. We totally detected 1,124 and 24 differentially expressed mRNAs and IncRNAs, respectively. The differentially expressed mRNAs were mainly associated with growth factor binding, protein K63-linked ubiquitination and cellular protein metabolic process; additionally, they were significantly enriched in the growth and development related pathways, including PPAR signaling pathway, vitamin B6 metabolism, glyoxylate and dicarboxylate metabolism. Combined analysis showed that the co-located differentially expressed IncRNA Lnc_002583 might positively influence the expression of the corresponding genes IFI44 and IFI44L, exerting co-regulative effects on Leiqiong cattle growth and development. Thus, we made the hypothesis that Lnc_002583, IFI44 and IFI44L might function synergistically to regulate the growth of Leiqiong cattle. This study provides a catalog of Leiqiong cattle liver mRNAs and IncRNAs, and will contribute to a better understanding of the molecular mechanism underlying growth regulataion.

\author{
Abbreviations \\ lncRNAs Long non-coding RNAs \\ RNA-seq RNA sequencing \\ DEGs Differentially expressed genes \\ ORF Open reading frame \\ FPKM Fragments per kilo base of exon per million fragments mapped \\ GRC Growth retardation cattle \\ NGC Normal growth cattle \\ GO Gene ontology \\ KEGG Kyoto Encyclopedia of Genes and Genomes
}

Beef is an excellent source of protein with lower level of lipid content and is rich in Fe, $\mathrm{P}$ and $\mathrm{Zn}$. Leiqiong cattle is one of the typical representative cattle in South China. It is heat-resistant, crude feed-resistant and has strong resistance to disease. However, Leiqiong cattle is small in size and low in meat production, which can not meet the needs of the market. Moreover, in the actual livestock production, some Leiqiong cattle will always have growth retardation. Currently, the causes of animal growth retardation are mainly divided into four categories, namely genetics, nutrition, disease, and feeding management. Animal growth retardation can cause a series of

\footnotetext{
${ }^{1}$ College of Animal Science, South China Agricultural University, Guangzhou 510642, GD, China. ${ }^{2}$ National Local Joint Engineering Research Center of Livestock and Poutry, South China Agricultural University, Guangzhou 510642, GD, China. ${ }^{3}$ These authors contributed equally: Lingxuan Kong and Guangbin

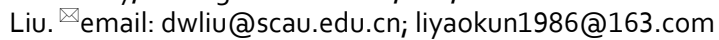


problems: the quality of the meat produced will decrease, the milk yield of dairy animals will decrease or milk production could cease, and the quality and grade of the fur of fur-producing animals will decrease. Thus, the economic benefits of animal production will be reduced when the animals suffer retardation.

Currently, research on animal growth retardation has focused on hormonal regulation and nutritional regulation. Japanese Black Cattle with growth retardation showed lower condentrations of serum growth hormone $(\mathrm{GH})$ and serum insulin like growth factor 1 (IGF1), suggesting defects in the GH-IGF1 axis ${ }^{1}$. Studies showed that GH deficient mice are $40 \%$ lighter than normal mice; IGF1 receptor deficient mice are $55 \%$ lighter than normal mice; meanwhile, these genetic deficient animals growth rate is significantly reduced, and the development of muscle and bone is also inhibited ${ }^{2}$. Additionally, it was suggested that daily feeding of $70 \mathrm{mg} / \mathrm{kg}$ body weight of cysteamine can significantly increase the daily weight gain and serum IGF1 levels of growing and finishing pigs, and can upregulate the expression of the growth hormone receptor (GHR), IGF1, IGF1 receptor (IGF1R), and insulin like growth factor binding protein 3 (IGFBP3) genes in multiple tissues ${ }^{3}$. The supplementation of sucrose can also improve the growth performance of slow-growing calves ${ }^{4}$. During feeding process, the growth retardation yak was supplemented with sucrose ( $1 \mathrm{~g} / \mathrm{kg}$ body weigh) twice a week; compared with growth-stasis cattle without sucrose supplementation, the body weight of sucrose-added yak was significantly increased and was similar to market weight; it was further depicted that the sucrose could induced rumen nipple development, which facilitated the compensatory growth of retardation yak. Genetic studies on growth retardation showed that if the homozygous form of the mouse Akt1 gene was disrupted, the mice would experience growth retardation ${ }^{5}$. Mice knocked out of both Akt1 and Akt2 showed weight loss, skeletal muscle atrophy, decreased fat deposition, and growth retardation ${ }^{6}$. When IGF1R was mutated, humans would suffer growth retardation ${ }^{7}$. The knockout of TMEFF2 gene could also result in growth retardation and severe loss of white adipose tissue in mice ${ }^{8}$. For growth retardation piglets, INS, IGF-1, and phosphorylation of AKT were significantly down-regulated ${ }^{9}$. Studies on animal growth retardation have focused on intrauterine growth retardation (IUGR). The IUGR phenomenon, for example, is widespread among multiparous animals and even among populations in many countries, with about $10 \%$ of babies born in the U.S. being affected by IUGR ${ }^{10}$. So far, animals such as rats ${ }^{11}$, mice ${ }^{10}$, guinea pigs $^{12}$, white rabbits ${ }^{13}$, sheep ${ }^{14}$, dogs ${ }^{15}$, and pigs $^{13}$ have been used to build IUGR models. IUGR not only affects the animal's birth weight and organ weight, but also affects the growth and development of the body and organs of the newborn after birth, such as the brain, small intestine, liver, muscles and so on ${ }^{9,16}$. It also affects the metabolism of animal liver nutrients such as carbohydrates, fatty acids, and amino acid metabolism ${ }^{16,17}$. Fatty acid beta oxidation in growth-stable newborn piglets is an important pathway for energy production, and some protein expressions are significantly down-regulated with fatty acid beta oxidation (EHHADH, GCDH and ACOX2), cholesterol synthesis (LSS), and lipid transport (APOE) $)^{18-20}$. Thus, we decided to study the molecular mechanisms that affect growth retardation in Leiqiong cattle. There is almost no research on the combination of long non-coding RNAs (lncRNAs) and growth retardation, and there is also little research on cattle growth retardation. The liver is the crucial metabolic organ associated with the growth response, and little is known about the mechanisms underlying growth retardation and development in cattle. Therefore, we focused on transcriptome analysis of the livers of growth retarded Leiqiong cattle and normal growth Leiqiong cattle to identify growthrelated mRNAs, lncRNAs, and regulatory pathways. We hypothesized that this approach would identify only the critical genes and pathways involved in the growth retardation response. The genes identified in this study could serve as candidates for breeding Leiqiong cattle with superior growth performance.

\section{Methods}

Animals and sample collection. The Leiqiong cattle (genus: Bos; species: Bos indicus) were born, raised, and maintained at the Guangdong Leiqiong cattle farm. In this study, all animals were healthy and received the same diet until they were slaughtered in adulthood (24 months). The experimental animals were divided into two groups: growth retardation cattle (GRC; the average body height was $95 \mathrm{~cm}$, and the average live weight before slaughter was $153.5 \mathrm{~kg}$ ) and normal growth cattle (NGC; the average body height was $106 \mathrm{~cm}$, and the average live weight before slaughter was $206.7 \mathrm{~kg}$ ). All the experimental animals were without any nutritional manipulations; they were simply spontaneously different in size and weight. Liver samples at the same predetermined site were collected from GRC and NGC ( $n=3$ at each group, female) and immediately frozen in liquid nitrogen for total RNA extraction after harvesting.

cDNA library preparation. We isolated the total RNA by using the TRIzol reagent (Invitrogen, Carlsbad, CA, USA) and DNase I (Qiagen, Beijing, China). Then, 1.5\% agarose gel electrophoresis was used to assess the quality of the purified RNA, confirming the absence of genomic DNA. The RNA integrity was estimated using an RNA Nano6000 Assay Kit and a Bioanalyzer 2100 system (Agilent Technologies, Santa Clara, CA, USA) ${ }^{21}$.

For the cDNA library contruction, $3 \mu \mathrm{g}$ of total RNA (the value of RNA integrity number was all larger than 7.0) was utilized as the input for each sample. Firstly, we discarded the ribosomal RNA (rRNA) through the Epicentre Ribo-zero rRNA Removal Kit (Epicentre, Madison, WI, USA), the rRNA-free residue was cleaned by ethanol precipitation. Next, sequencing libraries were constructed using the rRNA-deleted RNA with a NEBNext Ultra Directional RNA Library Prep Kit for Illumina (NEB, Ipswich, MA, USA) referring to the manufacturer's instructions. After fragmentation in NEBNext First Strand Synthesis Reaction Buffer $(5 \times)$, we subsequently synthesized the first strand and second strand cDNA. After adenylation of the $3^{\prime}$ ends of the DNA fragments, NEBNext Adaptors containing a hairpin loop structure were ligated to prepare for hybridization. The library fragments were purified using an AMPure XP system (Beckman Coulter, Beverly, MA, USA), preferentially selecting the cDNA fragments of 150-200 bp in length. Then, USER enzyme (NEB) was utilized to incubate with the size-selected, adaptor-ligated cDNA at $37^{\circ} \mathrm{C}$ for $15 \mathrm{~min}$ followed by $5 \mathrm{~min}$ at $95^{\circ} \mathrm{C}$. PCR amplification was then performed using Phusion High-Fidelity DNA polymerase, Universal PCR primers, and Index (X) Primer. 
Finally, the products were purified (AMPure XP system) and the library quality was assessed using the Agilent Bioanalyzer 2100 system $^{21}$.

Sequencing and transcriptome assembly. The libraries constructed were sequenced on an Illumina HiSeq 4000 platform and 150-bp paired-end reads were generated. After removing sequences containing adapters and the reads containing poly-N and low quality reads using in-house Perl scripts, clean data were obtained. All the downstream analyses were based on the high quality, clean data. To obtain the high quality reads, we performed the following filtering process: removing the reads containing more than $10 \%$ unknown nucleotides and the reads containing more than $50 \%$ low quality nucleotides with a Phred quality less than 20 . Mapping to the reference genome was the next step. Reads that passed the quality control were then mapped to the Bovine reference genome (Bos_taurus.UMD3.1; Ensemble release 94). An index of the reference genome was built using bowtie2 v2.2.8 and paired-end clean reads were aligned to the reference genome using HISAT2 (v2.0.4) ${ }^{22}$. HISAT2 was run with '-rna-strandness RF'; other parameters were set as default. Next, transcriptome assembly was performed using the mapped reads of each sample and StringTie (v1.3.1) ${ }^{23}$.

Prediction of differentially expressed long non-coding RNAs and protein-coding RNAs. Before screening, Cuffmerge was used to create the set of transcripts. Then, lncRNA screening was carried out using the following steps: Step 1: Select the number of exon $\geq 2$ transcripts; Step 2: From the results from step 1, select the transcripts with a length $>200$ bp; Step 3: Annotate the above transcripts using Cuffcompare software; Step 4: Calculate the expression level of each transcript using Cuffquant, and select transcripts with a fragments per kilo base of exon per million fragments mapped (FPKM) value $\geq 0.1$; Step 5: Coding potential screening. The coding potential of the transcript was predicted by three software modules: CNCI (Coding-Non-Coding-Index) (v2) ${ }^{24}$, CPC (Coding Potential Calculator) $(0.9-\mathrm{r} 2)^{25}$, and PFAM (Protein Families Database; Pfam Scan, v1.3) ${ }^{26,27}$; the intersection of transcripts without coding potential screened using the above three software modules with default parameters was predicted as the lncRNA dataset.

The Ballgown software was used to perform the straightforward linear-model-based differential expression analyses with the default statistical modeling framework ${ }^{23,28}$. Transcripts with an adjusted $P$ value $<0.05$ were assigned as differentially expressed.

Target gene prediction of the IncRNAs. For each lncRNA locus, the 100-kb regions upstream and downstream were chosen to screen for the cis-acting target genes using the UCSC Genome Bioinformatics tool, which were also named co-located genes.

GO and KEGG enrichment analysis. Gene Ontology (GO) enrichment analysis of differentially expressed genes (DEGs) or lncRNA target genes was implemented using the GOseq R package, in which gene length bias was corrected ${ }^{29}$. GO terms with corrected $P<0.05$ were considered significantly enriched for DEGs.

Kyoto Encyclopedia of Genes and Genomes (KEGG) is a database resource for understanding high-level functions and utilities of biological systems ${ }^{30}$, such as the cell, organism and ecosystem, from molecular-level information, especially large-scale molecular datasets generated by genome sequencing and other high-throughput experimental technologies (https://www.genome.jp/kegg/). We used KOBAS software to analyze the enrichment of DEGs or lncRNA target genes in KEGG pathways ${ }^{31}$.

Quantitative real-time reverse transcription polymerase chain reaction validation. For the quantitative real-time reverse transcription polymerase chain reaction (qRT-PCR) analysis, $1 \mu \mathrm{g}$ of total RNA was reverse transcribed using an RT reagent Kit with gDNA Eraser (Takara, Dalian, China) according to the manufacturer's protocol. The qRT-PCR reactions were performed on a StepOnePlus Real-Time PCR System (Life Technologies, Carlsbad, CA, USA) according to standard methods using Fast Start Universal SYBR Green Master (ROX) (Roche, Mannheim, Germany).

Ethics approval and consent to participate. The study was approved by the Ethics Committees of Laboratory Animal Center of South China Agricultural University (permit number: SYXK-2014-0136). All experiments were performed in accordance with South China Agricultural University guidelines.

\section{Results}

Read mapping. For RNA-seq data analysis, the proportion of the nucleotides with Q30 in the total nucleotides should be generally larger than $85 \%$; in our study, the proportion of each sample exceeded $91 \%$. In total, $89,186,385,77,069,075,80,073,002,91,238,926,94,484,701$ and 89,487,657 mapped reads were obtained from the clean data from the GRC1, GRC2, GRC3, NGC4, NGC5, and NGC6 libraries, respectively, and more than $94 \%$ were mapped to the Bovine reference genome (Table 1).

Enrichment analysis of differentially expressed mRNAs. We totally discovered 1,124 differentially expressed mRNA transcripts. Compared with the NGC group, 571 and 553 mRNAs were respectively upregulated and downregulated in the GRC group (Fig. 1A and Additional file 1). Systematic cluster analysis showed that the expression pattern of the differentially expressed mRNAs was obviously distinct between the two experimental groups (Fig. 1B). To further depict the biological function of the differentially expressed mRNAs, we performed GO and KEGG enrichment analysis. In total, $609 \mathrm{GO}$ terms with $P<0.05$ were found (Additional file 2); most of them were associated with growth and development, including growth factor binding, glucocor- 


\begin{tabular}{|l|l|l|l|l|l|l|}
\hline Sample & GRC1 & GRC2 & GRC3 & NGC4 & NGC5 & NGC6 \\
\hline Raw reads & $97,059,272$ & $84,633,280$ & $87,684,370$ & $99,905,226$ & $103,732,518$ & $97,539,080$ \\
\hline Clean reads & $94,190,850$ & $81,529,150$ & $85,130,830$ & $96,699,986$ & $100,268,668$ & $94,588,436$ \\
\hline Q30 (\%) & 93.39 & 93.39 & 91.98 & 93.36 & 93.48 & 93.44 \\
\hline GC content (\%) & 49.29 & 50.38 & 50.06 & 50.22 & 49.78 & 48.77 \\
\hline Total mapped & $\begin{array}{l}89,186,385 \\
(94.69 \%)\end{array}$ & $\begin{array}{l}77,069,075 \\
(94.53 \%)\end{array}$ & $\begin{array}{l}80,073,002 \\
(94.06 \%)\end{array}$ & $\begin{array}{l}91,238,926 \\
(94.35 \%)\end{array}$ & $\begin{array}{l}94,484,701 \\
(94.23 \%)\end{array}$ & $\begin{array}{l}89,487,657 \\
(94.61 \%)\end{array}$ \\
\hline
\end{tabular}

Table 1. Summary of clean reads mapped to the Bovine reference genome. GRC growth retardation cattle, $N G C$ normal growth cattle.

A

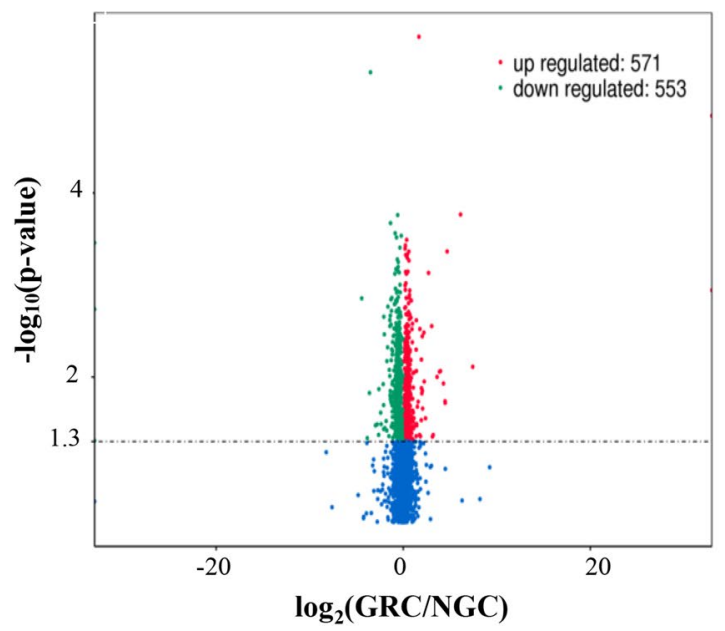

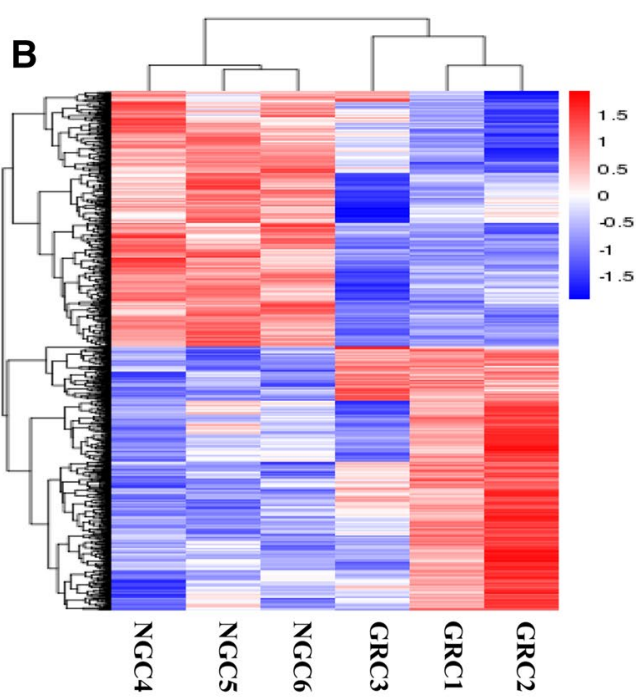

Figure 1. Analyses of differentially expressed mRNAs in the RNA sequencing (RNA-seq) libraries. (A) Volcano plot showing the overall distribution of the differential transcript or gene, with the threshold set to $\mathrm{q}<0.05$. Red: relatively high expression; Green: relatively low expression. (B) Hierarchical clustering analysis of mRNA expression profiles from 11 libraries with 1,124 differentially expressed mRNAs. Data are expressed as fragments per kilo base of exon per million fragments mapped (FPKM). Red: relatively high expression; Blue: relatively low expression.

ticoid receptor signaling pathway, "protein K63-linked ubiquitination, and cellular protein metabolic process (Table 2). Additionally, we detected 31 KEGG pathways significantly enriched by the differentially expressed mRNAs $(P<0.05)$ (Table 3 and Additional file 3$)$, several of which were related to growth and development, including PPAR signaling pathway, vitamin B6 metabolism, glyoxylate and dicarboxylate metabolism, and fatty acid metabolism. Of the top 50 DEGs, WIF1 was involved in the Wnt signaling pathway, and it also showed association with the regulation of fat cell differentiation, regulation of cell differentiation, and cellular developmental process; SDHAF4 was related to system development, organ development, single-organism developmental process and metabolic process.

Enrichment analysis of the differentially expressed IncRNAs. In our study, 24 lncRNAs were found differentially expressed (Fig. 2 and Additional file 4); the sequence of them was included in Additional file 5. Compared with NGC group, 18 lncRNAs were upregulated in the GRC group and 6 lncRNAs were downregulated (Table 4). For these differentially expressed lncRNAs, LNC_002093 and LNC_001379 were discovered 4 exons; LNC_002628, LNC_002005, LNC_000989, LNC_003503 and LNC_001876 showed 3 exons; all the other 17 lncRNAs had 2 exons, accounting for $70.83 \%$ of the differentially expressed lncRNAs (Additional file 4).

In total, we detected 114 protein-coding neighbors corresponding to the differentially expressed lncRNAs (Additional file 6). Based on the above targets of the lncRNAs, GO analysis revealed 561 significantly enriched GO terms $(P<0.05)$ (Additional file 7). According to the GO annotation, we screened the GO terms associated with growth and development, including positive regulation of cytolysis, succinate-semialdehyde dehydrogenase $(\mathrm{NAD}+)$ activity, cell chemotaxis, regulation of MAPK cascade, and positive regulation of smooth muscle cell proliferation (Table 5). Pathway analysis showed that the co-location genes were significantly enriched in 18 KEGG pathways $(P<0.05)$ (Additional file 8$)$, some of which were associated with growth and development, including Type I diabetes mellitus, chemokine signaling pathway and Toll-like receptor signaling pathway (Table 6). 


\begin{tabular}{|l|l|l|}
\hline GO terms & Number of genes & $P$ value \\
\hline Biological_process & 52 & $1.56 \times 10^{-6}$ \\
\hline GO:0019752: carboxylic acid metabolic process & 54 & $7.02 \times 10^{-6}$ \\
\hline GO:0006082: organic acid metabolic process & 53 & $7.74 \times 10^{-6}$ \\
\hline GO:0043436: oxoacid metabolic process & 53 & $3.55 \times 10^{-5}$ \\
\hline GO:0010033: response to organic substance & 95 & $4.22 \times 10^{-5}$ \\
\hline GO:0008152: metabolic process & 433 & $1.24 \times 10^{-5}$ \\
\hline GO:0044267: cellular protein metabolic process & 186 & $2.05 \times 10^{-4}$ \\
\hline GO:0030163: protein catabolic process & 46 & $6.09 \times 10^{-3}$ \\
\hline GO:0048522: positive regulation of cellular process & 168 & $1.57 \times 10^{-2}$ \\
\hline GO:0006950: response to stress & 117 & \\
\hline Cellular_component & 149 & $2.33 \times 10^{-12}$ \\
\hline Extracellular vesicular exosome & 337 & $4.53 \times 10^{-12}$ \\
\hline GO:0044444: cytoplasmic part & 223 & $5.89 \times 10^{-6}$ \\
\hline GO:0032991: macromolecular complex & 193 & $4.04 \times 10^{-5}$ \\
\hline GO:0043234: protein complex & 67 & $2.58 \times 10^{-3}$ \\
\hline GO:0012505: endomembrane system & 55 & $1.06 \times 10^{-2}$ \\
\hline GO:0005783: endoplasmic reticulum & \multicolumn{2}{|l}{} \\
\hline Molecular_function & 49 & $9.17 \times 10^{-5}$ \\
\hline GO:0016491: oxidoreductase activity & 255 & $1.35 \times 10^{-4}$ \\
\hline GO:0005515: protein binding & 81 & $1.53 \times 10^{-4}$ \\
\hline GO:0003723: RNA binding & 255 & $4.82 \times 10^{-4}$ \\
\hline GO:0003824: catalytic activity & 113 & $7.19 \times 10^{-4}$ \\
\hline GO:0036094: small molecule binding & 103 & $2.46 \times 10^{-3}$ \\
\hline GO:1901265: nucleoside phosphate binding & \multicolumn{2}{|l}{} \\
\hline
\end{tabular}

Table 2. Biological process enriched by the differentially expressed mRNAs.

\begin{tabular}{|l|c|l|}
\hline KEGG pathways & Number of genes & $\boldsymbol{P}$ value \\
\hline Proteasome & 19 & $3.01 \times 10^{-8}$ \\
\hline Metabolic pathways & 127 & $2.49 \times 10^{-4}$ \\
\hline Protein processing in endoplasmic reticulum & 26 & $8.49 \times 10^{-4}$ \\
\hline Vitamin B6 metabolism & 4 & $6.94 \times 10^{-3}$ \\
\hline Carbon metabolism & 16 & $7.80 \times 10^{-3}$ \\
\hline Glycine, serine and threonine metabolism & 9 & $8.18 \times 10^{-3}$ \\
\hline Tryptophan metabolism & 9 & $1.05 \times 10^{-2}$ \\
\hline Non-alcoholic fatty liver disease (NAFLD) & 21 & $1.32 \times 10^{-2}$ \\
\hline Pyrimidine metabolism & 15 & $1.38 \times 10^{-2}$ \\
\hline Glyoxylate and dicarboxylate metabolism & 6 & $1.71 \times 10^{-2}$ \\
\hline Alanine, aspartate and glutamate metabolism & 7 & $2.27 \times 10^{-2}$ \\
\hline PPAR signaling pathway & 11 & $2.37 \times 10^{-2}$ \\
\hline beta-Alanine metabolism & 6 & $4.28 \times 10^{-2}$ \\
\hline
\end{tabular}

Table 3. KEGG pathway enrichment analysis of differentially expressed mRNAs.

Combined analysis of the differentially expressed IncRNAs and differentially expressed mRNAs. Among the 114 protein-coding genes co-located with the differentially expressed lncRNAs, nine of them could be found in the DEGs list, including ILKAP, GLTPD2, PSMB6, IFI44, IFI44L, OSGIN1, CASP6, BOLA and B2M. For the 24 differentially expressed lncRNAs, LNC_001319 was located 43,587 bp upstream of PSMB6, which was enriched in the pathway of proteasome; GO analysis showed that PSMB6 was associated with cellular metabolic process, protein metabolic process, catalytic activity and organic substance catabolic process. LNC_001264 was located 79,489 bp upstream of OSGIN1; and OSGIN1 was related to metabolic process, catalytic activity, positive regulation of cellular process and positive regulation of biological process. LNC_002961 was located 24,399 bp upstream of CASP6, which was mainly involved in protein metabolic process and organic substance metabolic process. LNC_000301 was located 12,704 bp upstream of B2M; pathway analysis revealed that $\mathrm{B} 2 \mathrm{M}$ was enriched in antigen processing and presentation. 

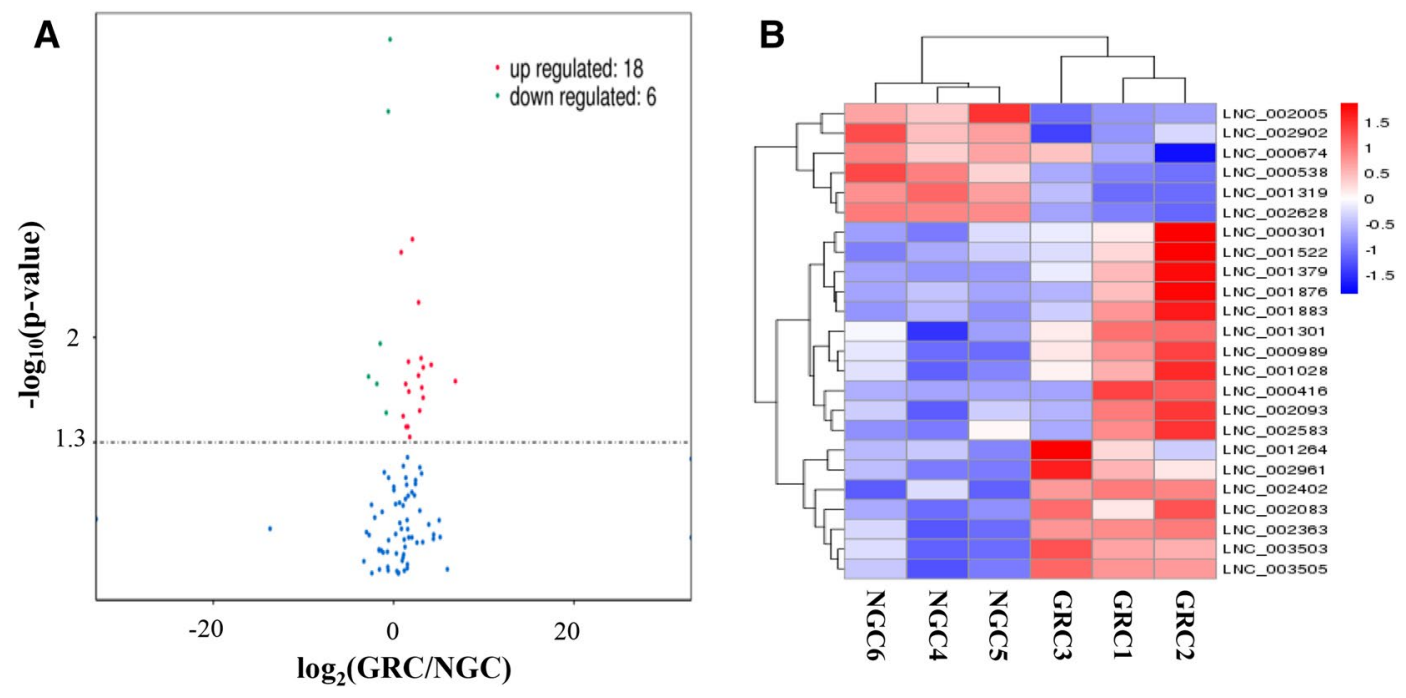

Figure 2. Analyses of differentially expressed lncRNAs in the RNA sequencing (RNA-seq) libraries. (A) Volcano plot showing the overall distribution of the differential transcripts or genes, with the threshold set to $P=0.05$. Red: relatively high expression; Green: relatively low expression. (B) Hierarchical clustering analysis of lncRNA expression profiles from 11 libraries with 24 differentially expressed lncRNAs. Data are expressed as fragments per kilo base of exon per million fragments mapped (FPKM). Red: relatively high expression; Blue: relatively low expression.

\begin{tabular}{|l|l|l|l|l|}
\hline Transcript ID & Gene ID & FPKM (GRC/NGC) & $\log _{2}$ (GRC/NGC) & P value \\
\hline LNC_002628 & XLOC_115691 & $5.82 / 7.64$ & -0.39314 & $1.04 \times 10^{-4}$ \\
\hline LNC_001319 & XLOC_060016 & $4.58 / 6.92$ & -0.59342 & $3.14 \times 10^{-4}$ \\
\hline LNC_002583 & XLOC_114076 & $5.04 / 1.19$ & 2.082334 & $2.22 \times 10^{-3}$ \\
\hline LNC_002093 & XLOC_093077 & $1.75 / 9.80$ & 0.8358590 & $2.71 \times 10^{-3}$ \\
\hline LNC_001264 & XLOC_057064 & $1.57 / 2.30$ & 2.76908 & $5.85 \times 10^{-3}$ \\
\hline LNC_000538 & XLOC_026579 & $6.44 / 1.80$ & -1.48464 & $1.10 \times 10^{-2}$ \\
\hline LNC_003505 & XLOC_162058 & $2.02 / 2.44$ & 3.048021 & $1.38 \times 10^{-2}$ \\
\hline LNC_002363 & XLOC_104219 & $6.43 / 2.06$ & 1.643864 & $1.45 \times 10^{-2}$ \\
\hline LNC_002961 & XLOC_132490 & $2.06 / 1.14$ & 4.167938 & $1.52 \times 10^{-2}$ \\
\hline LNC_001379 & XLOC_061866 & $4.57 / 4.70$ & 3.283323 & $1.59 \times 10^{-2}$ \\
\hline LNC_001883 & XLOC_086039 & $2.01 / 2.98$ & 2.757798 & $1.79 \times 10^{-2}$ \\
\hline LNC_002902 & XLOC_130325 & $8.01 / 5.51$ & -2.78255 & $1.82 \times 10^{-2}$ \\
\hline LNC_000416 & XLOC_020667 & $1.61 / 1.40$ & 6.847665 & $1.96 \times 10^{-2}$ \\
\hline LNC_002005 & XLOC_090526 & $1.21 / 4.40$ & -1.85589 & $2.04 \times 10^{-2}$ \\
\hline LNC_002402 & XLOC_106316 & $5.28 / 2.08$ & 1.340555 & $2.04 \times 10^{-2}$ \\
\hline LNC_000989 & XLOC_045297 & $1.79 / 2.04$ & 3.126715 & $2.16 \times 10^{-2}$ \\
\hline LNC_001028 & XLOC_046713 & $3.01 / 9.33$ & 1.688786 & $2.29 \times 10^{-2}$ \\
\hline LNC_003503 & XLOC_162058 & $2.88 / 2.98$ & 3.272133 & $2.52 \times 10^{-2}$ \\
\hline LNC_002083 & XLOC_092862 & $1.86 / 2.51$ & 2.890866 & $3.08 \times 10^{-2}$ \\
\hline LNC_000674 & XLOC_032076 & $4.33 / 7.64$ & -0.81978 & $3.17 \times 10^{-2}$ \\
\hline LNC_001301 & XLOC_058880 & $4.53 / 2.17$ & 1.060875 & $3.34 \times 10^{-2}$ \\
\hline LNC_001522 & XLOC_067835 & $1.74 / 8.52$ & 1.580438 & $3.93 \times 10^{-2}$ \\
\hline LNC_001876 & XLOC_085744 & $1.39 / 5.31$ & 1.391823 & $3.94 \times 10^{-2}$ \\
\hline LNC_000301 & XLOC_015845 & $5.97 / 1.73$ & 1.788629 & $4.60 \times 10^{-2}$ \\
\hline & & & & \\
\hline
\end{tabular}

Table 4. The 24 differentially expressed lncRNAs in the liver of Leiqiong cattle. FPKM fragments per kilo base of exon per million fragments mapped, GRC growth retardation cattle, NGC normal growth cattle. 


\begin{tabular}{|l|l|l|}
\hline GO terms & Number of genes & $P$ value \\
\hline Biological_process & 6 & $5.87 \times 10^{-6}$ \\
\hline GO:0048247: lymphocyte chemotaxis & 6 & $9.41 \times 10^{6}$ \\
\hline GO:0034341: response to interferon-gamma & 7 & $1.61 \times 10^{-5}$ \\
\hline GO:0070374: positive regulation of ERK1 and ERK2 cascade & 8 & $3.36 \times 10^{-5}$ \\
\hline GO:0043410: positive regulation of MAPK cascade & 8 & $1.22 \times 10^{-4}$ \\
\hline GO:0071347: cellular response to interleukin-1 & 5 & $1.70 \times 10^{-4}$ \\
\hline GO:0070098: chemokine-mediated signaling pathway & 5 & $2.94 \times 10^{-4}$ \\
\hline GO:0034612: response to tumor necrosis factor & 6 & $5.50 \times 10^{-4}$ \\
\hline GO:0019637: organophosphate metabolic process & 10 & $5.72 \times 10^{-4}$ \\
\hline GO:0006152: purine nucleoside catabolic process & 6 & $6.25 \times 10^{-4}$ \\
\hline GO:0043065: positive regulation of apoptotic process & 5 & $1.25 \times 10^{-3}$ \\
\hline GO:1901136: carbohydrate derivative catabolic process & 6 & \\
\hline Cellular_component & \multicolumn{2}{|l}{} \\
\hline GO:0042612: MHC class I protein complex & 3 & $2.43 \times 10^{-4}$ \\
\hline GO:0044420: extracellular matrix part & 3 & $2.81 \times 10^{-3}$ \\
\hline GO:0005615: extracellular space & 12 & $7.18 \times 10^{-3}$ \\
\hline GO:0005578: proteinaceous extracellular matrix & 3 & $3.76 \times 10^{-2}$ \\
\hline Molecular_function & 7 & $3.17 \times 10^{-7}$ \\
\hline GO:0008009: chemokine activity & 7 & $4.93 \times 10^{-7}$ \\
\hline GO:0042379: chemokine receptor binding & 5 & $1.85 \times 10^{-6}$ \\
\hline GO:0042605: peptide antigen binding & 5 & $8.09 \times 10^{-6}$ \\
\hline GO:0001664: G-protein coupled receptor binding & $5.60 \times 10^{-5}$ \\
\hline GO:0048020: CCR chemokine receptor binding & \multicolumn{2}{|l}{} \\
\hline
\end{tabular}

Table 5. Gene ontology (GO) enrichment analysis of the protein-coding genes co-located with the differentially expressed cis-acting lncRNAs.

\begin{tabular}{|l|l|l|}
\hline KEGG pathway & Number of genes & $P$ value \\
\hline Herpes simplex infection & 9 & $6.07 \times 10^{-5}$ \\
\hline Antigen processing and presentation & 5 & $5.70 \times 10^{-4}$ \\
\hline Viral myocarditis & 5 & $3.96 \times 10^{-4}$ \\
\hline Type I diabetes mellitus & 4 & $1.23 \times 10^{-3}$ \\
\hline Chemokine signaling pathway & 7 & $1.43 \times 10^{-3}$ \\
\hline Autoimmune thyroid disease & 4 & $2.28 \times 10^{-3}$ \\
\hline Linoleic acid metabolism & 3 & $4.09 \times 10^{-3}$ \\
\hline Toll-like receptor signaling pathway & 4 & $1.45 \times 10^{-2}$ \\
\hline
\end{tabular}

Table 6. Kyoto Encyclopedia of Genes and Genomes (KEGG) pathway enrichment analysis of the proteincoding genes co-located with the differentially expressed cis-acting lncRNAs.

Validation of differentially expressed IncRNAs and mRNAs. We randomly selected six differentially expressed lncRNAs and six differentially expressed mRNAs for qRT-PCR analysis. Results demonstrated that the gene expression trend in the two experimental groups was consistent between RNA-Seq and qRT-PCR method, although the fold change of the expression level, to some extent, differed between the two sets of data (Fig. 3).

\section{Discussion}

Liver is an important organ for nutrient absorption and metabolism. However, in the liver of growth retardation fetuses, there are always disorders of growth and development and nutrient metabolism ${ }^{32-35}$. Therefore, in the present study, we performed transcriptome sequencing for the liver of Leiqiong cattle with growth retardation and those with normal growth and analyzed the differentially expressed mRNAs and lncRNAs to clarify their roles in growth and development. In this study, our results provided a valuable catalog of functional lncRNAs and mRNAs associated with growth and development.

Through RNA-Seq, we totally identified 1,124 differentially expressed mRNAs and 24 differentially expressed lncRNAs between GRC and NGC experimental groups, which may have specific biological roles in growth retardation in Leiqiong cattle. Using qRT-PCR, twelve randomly selected differentially expressed lncRNAs and mRNAs transcripts were validated, and the results were found to be consistent with the RNA-seq data. Among 

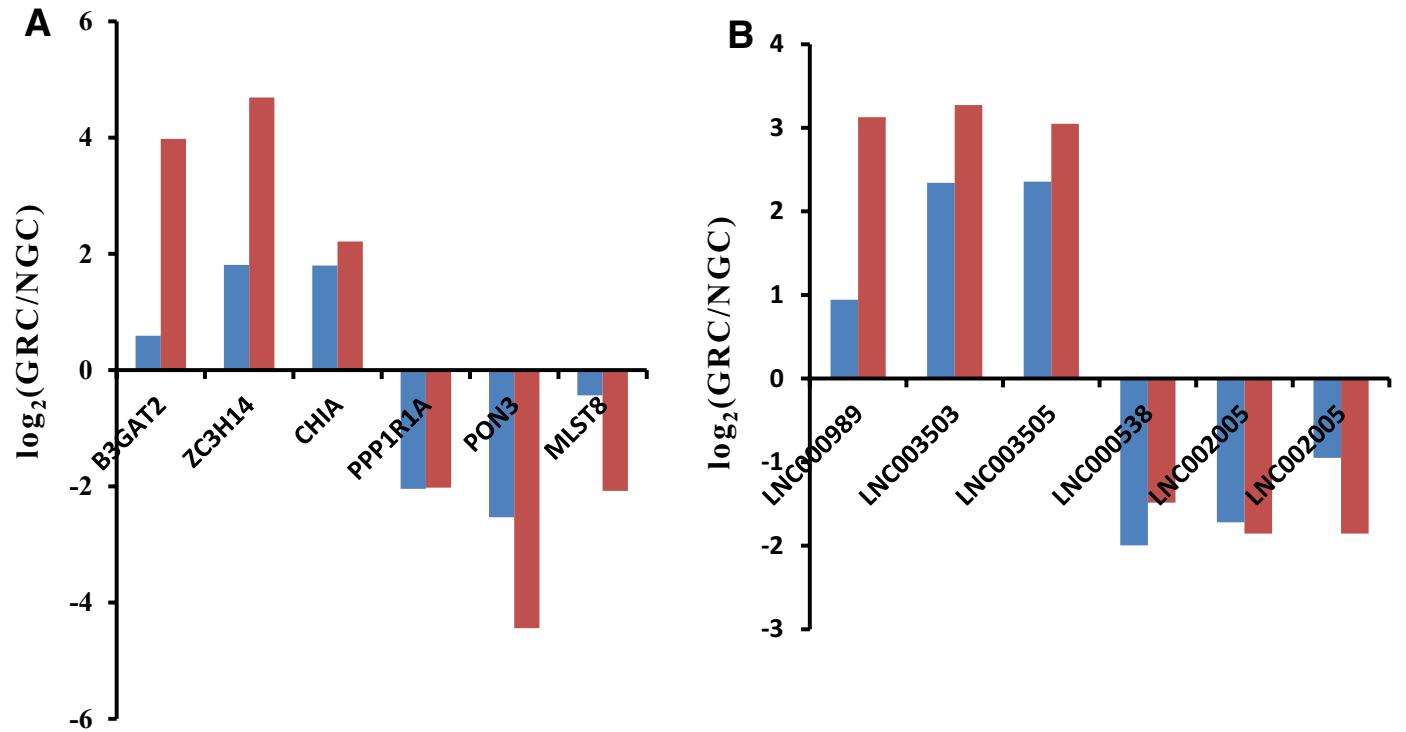

Figure 3. Validation of six differentially expressed mRNAs and long non-coding RNAs (lncRNAs) using quantitative real-time reverse transcription PCR (qRT-PCR). (A) The qRT-PCR results of differentially expressed mRNAs were compared with the RNA sequencing (RNA-seq) data. Red: RNA-seq; Blue: qRT-PCR. (B) The qRT-PCR results of differentially expressed lncRNAs were compared with the RNA-seq data. Red: RNAseq; Blue: qRT-PCR.

the identified DEGs, wnt inhibitor factor 1 (WIF1) and encoding succinate dehydrogenase complex assembly factor 4 (SDHAF4) are significantly differently expressed. WIF1 is an extracellular inhibitor of the wingless signaling pathway that binds directly to Wnt proteins, thereby inhibiting the transmission of Wnt signaling. The Wnt signaling pathway transmits a growth stimulation signal, and the abnormal activation of the pathway can cause abnormal proliferation and differentiation of cells and cause tumor formation ${ }^{36}$. The WIF1 gene also appears as a paternal gene-expressing imprinted gene in human embryonic trophoblasts ${ }^{37,38}$. In our study, the expression of WIF1 gene increased in the GRC group, biological analysis results revealed that WIF1 was engaged in the GO terms including "regulation of fat cell differentiation", "regulation of cell differentiation", "cellular developmental process". Studies demonstrated that SDHAF4 gene could promote mitochondrial succinate dehydrogenase activity and prevent neurodegeneration ${ }^{39}$. In livestock and poultry research, the biological function of SDHAF4 has been rarely reported. In our study, the expression of SDHAF4 decreased in the GRC group, and the bioinformatic analyses revealed that SDHAF4 was engaged in the GO terms including "cellular metabolic process", "succinate dehydrogenase (ubiquinone) activity", "organ development", and "mitochondrial respiratory chain complex assembly". Thus, we made the hypothesis that SDHAF4 might play roles in regulating the growth and development of Leiqiong cattle; however, the underlying mechanism still requires further investigation. The ACO2 gene can promote the biosynthesis of lysine, generate ATP by participating in the tricarboxylic acid cycle, affect the proportion of muscle fibers, and regulate muscle growth and development ${ }^{40,41}$. For the genes B3GALT1 and UPP2 that we found to be extremely different, there are no reports related to the growth and development of the body, which requires further cytological validation. In our study, the expression of ACO2 and UPP2 were decreased in the GRC group and the expression of B3GALT1 was increased. Through bioinformatics analysis, we found that ACO2, B3GALT1 and UPP2 are mainly involved in Metabolic pathways, Carbon metabolism, Citrate cycle (TCA cycle), Glyoxylate and dicarboxylate metabolism, Biosynthesis of amino acids. The JUNB gene product (JunB proto-oncogene, AP-1 transcription factor subunit) has been described as a growth-inhibiting protein, and JUNB deficient mice showed a retarded growth and a reduction in adipose tissue $e^{42}$. The expression of transcription factor JUNB was significantly down-regulated in the model of muscle atrophy induced by diabetes, denervation and starvation. JUNB also plays an important role in regulating the synthesis and degradation of skeletal muscle proteins. It has been reported that knocking down JUNB in mice can significantly reduce the cross-sectional area of muscle fibers, while overexpression can significantly increase the cross-sectional area of muscle fibers and promote muscle hypertrophy in mice. The main reason for muscle hypertrophy is that JUNB promotes protein synthesis and inhibits protein degradation ${ }^{43}$. In our study, the expression of JUNB gene increased in the GRC group, biological analysis results revealed that JUNB was engaged in the KEGG pathway including "osteoclast differentiation", "TNF signaling pathway". EGFR is a member of ErbB family. It has tyrosine kinase activity and is an important transmembrane receptor. EGFR was positively correlated with the development of central nervous system and the growth ability of cultured neurons in vitro. EGFR is activated by ligands to initiate intracellular signal transduction. It regulates transcription of transcription factor-activated genes through cascades of binding proteins and enzymes in cytoplasm, and directly participates in cell migration, adhesion, proliferation, differentiation and apoptosis. EGFR is expressed in chondrocyte and can promote chondrocyte proliferation by binding with ligands ${ }^{44,45}$. Overexpression of EGFR in mice can accelerate the proliferation and growth of osteoblasts ${ }^{46}$ and the proliferation of osteoblasts is impaired after knockout ${ }^{47}$. In our study, the expression of EGFR gene 
decreased in the GRC group, biological analysis results revealed that EGFR was engaged in the KEGG pathway including "regulation of actin cytoskeleton", "PI3K-Akt signaling pathway", "ErbB signaling pathway", "MAPK signaling pathway". KEGG pathway enrichment analysis showed that PPAR signaling pathway was significantly enriched by the DEGs ACOX2, SCD5, CPT1A, PPARa, ACOX1, RXRB, PCK2, SLC27A5, CPT2, EHHADH and RXRA, playing key roles in the regulation of multiple types of cells metabolism process. As a key member of this pathway, PPARa is involved in lipid metabolism in the liver and muscle, and is a key regulator of hepatocytes growth and metabolism; it has been demonstrated that PPARa could be utilized as a candidate gene for markerassisted selection for growth in cattle ${ }^{48-50}$. CPT1A gene is reported to be involved in regulating the growth traits in goat ${ }^{51}$. RXRA could influence cells proliferation and survival, influencing animals immune reaction ${ }^{52}$. PCK2 is suggested to play certain roles in modifying the function of liver and the development of muscle $\mathrm{e}^{53,54}$. Thus, we hypothesized that these above genes were involved in animals' growth and development process; however, the underlying mechanism still required further investigation. In the next work, we will further verify the function of the genes found in the above on the cells.

Transcriptional regulation could be affected by the activities of non-coding RNA (ncRNA) and transcription factors. Only about $2 \%$ of the mammalian genome is transcribed as proteins; $75-90 \%$ is transcribed as ncRNAs, the vast majority of which were $\operatorname{lncRNAs} s^{55}$. In human and mouse, lncRNAs have been reported to potentially regulate muscle growth and differentiation ${ }^{56-58}$. In the present study, we totally identified 3,705 lncRNAs; 24 of them were differentially expressed in pairwise comparison between GRC and NGC groups, which might have specific biological roles in regulating animals growth and development. Studies have demonstrated that lncRNAs could influence the myoblast proliferation and differentiation through regulating the growth related genes; additonally, lncRNAs could significantly affect miRNA biology by acting as a precursor for miRNAs, directly binding to and sequestering miRNAs, or indirectly interfering with miRNA expression and regulation, through which the animal growth and development process might be regulated ${ }^{59,60}$. And, lncRNAs might work as competing endogenous RNA (ceRNA) model, binding to and sequestering miRNAs to prevent their target transcript degradation ${ }^{61,62}$; however, the ceRNA hypothesis remains controversial. In the present study, our identified lncRNAs and their functions were predicted by bioinformatics method, thus further functional experiment should be performed for the validation, facilitating the enrichment of the functional annotation of the identified lncRNAs. For the differentially expressed lncRNAs, most of them were discovered two exons, accounting for $70.83 \%$; just two lncRNAs showed the highest number of the exon, which was four. These lncRNAs were spliced with fewer exons (2-4), which was consistent with previous studies ${ }^{63}$. Their lower number of exons compared to mRNAs could be caused by the weaker expression of lncRNAs, and this made their structure more diffcult to verify ${ }^{64}$. GO analysis showed that the differentially expressed lncRNAs targets were associated with smooth muscle cell proliferation, cell chemotaxis, and regulation of MAPK cascade. The target genes (MAPK8, RPS6KA2) of Lnc_002363 and Lnc_003503 were significantly enriched in the MAPK signaling pathway; this pathway is related to embryonic development, cell proliferation, division, inflammation, cancer and so on ${ }^{65,66}$. Therefore, in addition to mRNAs, the differentially expressed lncRNAs reported here could be considered as important novel regulatory factors involved in the growth and development process in Leiqiong cattle. Among the 24 screened lncRNAs, Lnc_002583 was found located between the differentially expressed genes IFI44 and IFI44L. IFI44 is a kind of interferon-induced gene. The increase of IFI44 expression induces interferon response and inhibits the expression of MSTN gene. Most interferon-inducible genes can regulate cell growth. Some studies have found that IFI44 can inhibit cell proliferation. By transfecting IFI44 into melanoma cells, it was found that IFI44 could reduce the sensitivity of cells to IFN-alpha and inhibit cell growth ${ }^{67}$. The expression of IFI44L was up-regulated in synovial tissue of patients with systemic lupus erythematosus ${ }^{68}$. In order to study the role of IFI44 and IFI44L in the growth and development of Leiqiong cattle, GO enrichment analysis was carried out. The results showed that they were associated with small molecule binding, nucleotide binding, purine ribonucleoside binding, organic cyclic compound binding, GTP binding, viral defense response, stress response and immune system process. Lnc_002583, IFI44 and IFI44L were all upregulated in GRC group; due to the potential cis-regulatory effect, Lnc_002583 might positively influence the expression of IFI44 and IFI44L, exerting co-regulative effects on Leiqiong cattle growth and development. Our identified differentially expressed lncRNAs were all novel lncRNAs, they might act as ceRNAs to regulate animals growth; their regulatory effects in other species are still uncertain, which needs further verification. For the long non-coding RNAs related to the growth and development of the above screening, and the regulation relationship with the target genes, further experiment should be performed to verify the function and explore their regulation mechanism on the growth and development of Leiqiong cattle.

\section{Conclusions}

The present study provided a systematic description of the changes in mRNAs and lncRNAs in Leiqiong cattle under the condition of growth retardation. The data was helpful for further investigation of the lncRNAs function. Generally, our results contributed to basic information to elucidate the mechanism associated the regulation of growth retardation in Leiqiong cattle at molecular level.

\section{Data availability}

Our sequencing data is being loaded to the sequence read archive (SRA) of NCBI. Once the submission ID was obtained, we would include it in our manuscript.

Received: 2 April 2020; Accepted: 10 August 2020

Published online: 31 August 2020 


\section{References}

1. Kitagawa, H. et al. Serum growth hormone and insulin-like growth factor-1 concentrations in Japanese black cattle with growth retardation. J. Vet. Med. Sci. 63(2), 167-170 (2001).

2. Lupu, F., Terwilliger, J. D., Lee, K., Segre, G. V. \& Efstratiadis, A. Roles of growth hormone and insulin-like growth factor 1 in mouse postnatal growth. Dev. Biol. 229(1), 141-162 (2001).

3. Liu, G., Wei, Y., Wang, Z., Wu, D. \& Zhou, A. Effects of dietary supplementation with cysteamine on growth hormone receptor and insulin-like growth factor system in finishing pigs. J. Agric. Food Chem. 56(13), 5422-5427 (2008).

4. Sato, T., Hidaka, Y. \& Kamimura, S. Sugar supplementation stimulates growth performance in calves with growth retardation. J. Vet. Med. Sci. 72(1), 29-33 (2010).

5. Chen, W. S. et al. Growth retardation and increased apoptosis in mice with homozygous disruption of the Akt1 gene. Genes Dev 15(17), 2203-2208 (2001).

6. Peng, X. D. et al. Dwarfism, impaired skin development, skeletal muscle atrophy, delayed bone development, and impeded adipogenesis in mice lacking Akt1 and Akt2. Genes Dev. 17(11), 1352-1365 (2003).

7. Abuzzahab, M. J. et al. IGF-I receptor mutations resulting in intrauterine and postnatal growth retardation. N. Engl. J. Med. 349(23), 2211-2222 (2003)

8. Chen, T. R. et al. Generation and characterization of Tmeff2 mutant mice. Biochem. Biophys. Res. Commun. 425(2), 189-194 (2012).

9. Wang, Y. et al. Inhibitory effect of adenovirus-mediated siRNA-targeting BMPR-IB on UHMWPE-induced bone destruction in the murine air pouch model. Connect. Tissue Res. 53(6), 528-534 (2012).

10. Buchmiller-Crair, T. L. et al. Delayed disaccharidase development in a rabbit model of intrauterine growth retardation. Pediatr. Res. 50(4), 520-524 (2001).

11. Hayashi, T. T. \& Dorko, M. E. A rat model for the study of intrauterine growth retardation. Am. J. Obstet. Gynecol. 158(5), 1203-1207 (1988)

12. Rockwell, L. C., Keyes, L. E. \& Moore, L. G. Chronic hypoxia diminishes pregnancy-associated DNA synthesis in guinea pig uteroplacental arteries. Placenta 21(4), 313-319 (2000).

13. Bassan, H. et al. Experimental intrauterine growth retardation alters renal development. Pediatr. Nephrol. 15(3-4), 192-195 (2000).

14. Phillips, I. D. et al. Restriction of fetal growth has a differential impact on fetal prolactin and prolactin receptor mRNA expression. J. Neuroendocrinol. 13(2), 175-181 (2010).

15. Kliegman, R. M. Alterations of fasting glucose and fat metabolism in intrauterine growth-retarded newborn dogs. Am. J. Physiol. 256(3 Pt 1), E380-385 (1989).

16. Wang, J. J. et al. Intrauterine growth restriction affects the proteomes of the small intestine, liver, and skeletal muscle in newborn pigs. J. Nutr. 138(1), 60-66 (2008).

17. Liu, C. et al. Intrauterine growth restriction alters the hepatic proteome in fetal pigs. J. Nutr. Biochem. 24(6), 954-959 (2013).

18. Yeh, C. S. et al. Fatty acid metabolism pathway play an important role in carcinogenesis of human colorectal cancers by MicroarrayBioinformatics analysis. Cancer Lett. 233(2), 297-308 (2006).

19. Villagra, A. et al. Histone deacetylase 3 down-regulates cholesterol synthesis through repression of lanosterol synthase gene expression. J. Biol. Chem. 282(49), 35457-35470 (2007).

20. Mahley, R. W. Apolipoprotein E: cholesterol transport protein with expanding role in cell biology. Science 240(4852), 622-630 (1988).

21. Li, Y. K. et al. Heat stress-responsive transcriptome analysis in the liver tissue of Hu sheep. Genes 10(5), 395 (2019).

22. Oh, J. et al. Temporal stability of the human skin microbiome. Cell 165(4), 854-866 (2016).

23. Pertea, M., Kim, D., Pertea, G. M., Leek, J. T. \& Salzberg, S. L. Transcript-level expression analysis of RNA-seq experiments with HISAT, StringTie and Ballgown. Nat. Protoc. 11(9), 1650-1667 (2016).

24. Sun, L. et al. Utilizing sequence intrinsic composition to classify protein-coding and long non-coding transcripts. Nucleic Acids Res. 41(17), e166 (2013).

25. Lei, K. et al. CPC: assess the protein-coding potential of transcripts using sequence features and support vector machine. Nucleic Acids Res. 35(Web Server issue), W345 (2007).

26. Finn, R. D. et al. The Pfam protein families database: towards a more sustainable future. Nucleic Acids Res. 44(D1), D279-285 (2016).

27. Ding, W. et al. Domain-oriented functional analysis based on expression profiling. BMC Genom. 3(1), 32 (2002).

28. Xue, D. Q. et al. Transcriptome analysis of the Cf-12-mediated resistance response to Cladosporium fulvum in tomato. Front. Plant Sci. 2016, 7 (2012)

29. Young, M. D., Wakefield, M. J., Smyth, G. K. \& Oshlack, A. Gene ontology analysis for RNA-seq: accounting for selection bias. Genome Biol. 11(2), R14 (2010).

30. Kanehisa, M. et al. KEGG for linking genomes to life and the environment. Nucleic Acids Res. 36(Database issue), D480-D484 (2008).

31. Tao, C. Automated genome annotation and pathway identification using the KEGG Orthology (KO) as a controlled vocabulary. Bioinformatics 21(19), 3787-3793 (2005).

32. Peterside, I. E., Selak, M. A. \& Simmons, R. A. Impaired oxidative phosphorylation in hepatic mitochondria in growth-retarded rats. Am. J. Physiol. Endocrinol. Metab. 285(6), E1258-1266 (2003).

33. Thorn, S. R. et al. Intrauterine growth restriction increases fetal hepatic gluconeogenic capacity and reduces messenger ribonucleic acid translation initiation and nutrient sensing in fetal liver and skeletal muscle. Endocrinology 150(7), 3021-3030 (2009).

34. Cianfarani, S. et al. Effect of intrauterine growth retardation on liver and long-term metabolic risk. Int. J. Obes. 36(10), 1270-1277 (2012).

35. Liu, J. et al. Effects of intrauterine growth retardation and maternal folic acid supplementation on hepatic mitochondrial function and gene expression in piglets. Arch. Anim. Nutr. 66(5), 357-371 (2012)

36. Polakis, P. Wnt signaling and cancer. Genes Dev. 14, 1837-1851 (2000).

37. Guilleret, I. et al. Imprinting of tumor-suppressor genes in human placenta. Epigenetics 4(1), 62-68 (2009).

38. Hsieh, J. C. et al. A new secreted protein that binds to Wnt proteins and inhibits their activities. Nature 398(6726), 431 (1999).

39. Vranken, J. G. et al. SDHAF4 promotes mitochondrial succinate dehydrogenase activity and prevents neurodegeneration. Cell Metab. 20(2), 241-252 (2014).

40. Fazius, F., Shelest, E. \& Gebhardt, P. et al. The fungal a-aminoadipate pathway for lysine biosynthesis requires two enzymes of the aconitase family for the isomerization of homocitrate to homoisocitrate. Mol. Microbiol. 86(6), 1508-1530 (2012).

41. Priyadarshini, Y. \& Natarajan, K. Reconfiguration of transcriptional control of lysine biosynthesis in candida albicans involves a central role for the Gcn 4 transcriptional activator. mSphere. 1(1), 1-14 (2016).

42. Montserrat, P. et al. Adipose triglyceride lipase and hormone-sensitive lipase are involved in fat loss in JunB-deficient mice. Endocrinology 152(7), 2678-2689 (2011).

43. Raffaello, A. et al. JunB transcription factor maintains skeletal muscle mass and promotes hypertrophy. J. Cell Biol. 191(1), 101-113 (2010).

44. Davideau, J. L., Sahlberg, C., Thesleff, I. \& Berdal, A. EGF receptor expression in mineralized tissues: an in situ hybridization and immunocytochemical investigation in rat and human mandibles. Connect. Tissue Res. 32(1-4), 47-53 (1995). 
45. Nawachi, K. et al. Tyrosine kinase-type receptor ErbB4 in chondrocytes: interaction with connective tissue growth factor and distribution in cartilage. FEBS Lett. 528(1-3), 109-113 (2002).

46. Chien, H. H., Lin, W. L. \& Cho, M. I. Down-regulation of osteoblastic cell differentiation by epidermal growth factor receptor. Calcif. Tissue Int. 67(2), 141-150 (2000).

47. Maria, S. et al. Mice humanised for the EGF receptor display hypomorphic phenotypes in skin, bone and heart. Development 130(19), 4515 (2003).

48. Chen, S. \& Li, Y. Current perspectives on the roles of PPARa in nonalcoholic fatty liver disease and hepatocarcinogenesis. Zhonghua gan zang bing za zhi = Zhonghua ganzangbing zazhi/Chin. J. Hepatol. 22(9), 718-720 (2014).

49. Zhang, J. Q. et al. Relationship between PPARa mRNA expression and mitochondrial respiratory function and ultrastructure of the skeletal muscle of patients with COPD. Bioengineered 8(6), 723-731 (2017).

50. Chen, N. B. et al. Chinese yellow cattle PPARA gene: analyses of expression, polymorphism and trait association. Czech. J. Anim. Sci. 63(12), 473-482 (2018).

51. Li, W. Y., Liu, Y., Gao, C. F., Lan, X. Y. \& Wu, X. F. A novel duplicated insertion/deletion (InDel) of the CPT1a gene and its effects on growth traits in goat. Anim. Biotechnol. (48), 1-9 (2019).

52. Stephensen, C. B., Borowsky, A. D. \& Lloyd, K. C. Disruption of Rxra gene in thymocytes and T lymphocytes modestly alters lymphocyte frequencies, proliferation, survival and T helper type 1/type 2 balance. Immunology 121(4), 484-498 (2007).

53. Gentili, S., Morrison, J. L. \& McMillen, I. C. Intrauterine growth restriction and differential patterns of hepatic growth and expression of IGF1, PCK2, and HSDL1 mRNA in the sheep fetus in late gestation. Biol. Reprod. 80(6), 1121-1127 (2009).

54. Loczenski-Brown, D. M. et al. Effect of adeno-associated virus (AAV)-mediated overexpression of PEPCK-M (Pck2) on Clenbuterol-induced muscle growth. PLoS ONE 14(6), e0218970.52 (2019).

55. Derrien, T. et al. Knowles DG: The GENCODE v7 catalog of human long noncoding RNAs: analysis of their gene structure, evolution, and expression. Genome Res. 22(1775), 1789 (2012).

56. Monica, B. et al. Novel long noncoding RNAs (lncRNAs) in myogenesis: a miR-31 overlapping lncRNA transcript controls myoblast differentiation. Mol. Cell. Biol. 35(4), 728 (2015).

57. Legnini, I., Morlando, M., Mangiavacchi, A., Fatica, A. \& Bozzoni, I. A feedforward regulatory loop between HuR and the long noncoding RNA linc-MD1 controls early phases of myogenesis. Mol. Cell 53(3), 506-514 (2014).

58. Mueller, A. C. et al. MUNC, a long noncoding RNA that facilitates the function of MyoD in skeletal myogenesis. Mol. Cell. Biol. 35(3), 498-513 (2015).

59. Yue, Y. et al. A lncRNA promotes myoblast proliferation by up-regulating GH1. In vitro cellular \& developmental biology. Animal 53(8), 699-705 (2017).

60. Sahu, A., Singhal, U. \& Chinnaiyan, A. M. Long noncoding RNAs in cancer: from function to translation. Trends Cancer 1(2), 93-109 (2015)

61. Salmena, L., Poliseno, L., Tay, Y., Kats, L. \& Pandolfi, P. P. A ceRNA hypothesis: the Rosetta Stone of a hidden RNA language?. Cell 146(3), 353-358 (2011).

62. Tay, Y., Rinn, J. \& Pandolfi, P. P. The multilayered complexity of ceRNA crosstalk and competition. Nature 505(7483), 344-352 (2014).

63. Sylvain, F. et al. Transcriptome and chromatin structure annotation of liver, CD4 and CD8 T cells from four livestock species. Biorxiv Prepr. https://doi.org/10.1101/316091 (2018).

64. Lagarde, J. et al. Extension of human lncRNA transcripts by RACE coupled with long-read high-throughput sequencing (RACESeq). Nat. Commun. 7, 12339 (2016).

65. Wagner, E. F. \& Nebreda, A. R. Signal integration by JNK and p38 MAPK pathways in cancer development. Nat. Rev. Cancer 9(8), 537-549 (2009).

66. Roux, P. P. \& Blenis, J. ERK and p38 MAPK-activated protein kinases: a family of protein kinases with diverse biological functions. Microbiol. Mol. Biol. Rev. 68(2), 320-344 (2004).

67. Hallen, L. C. et al. Antiproliferative activity of the human IFN-alpha-inducible protein IFI44. J. Interferon Cytokine Res. 27(8), $675-680$ (2007).

68. Nzeusseu Toukap, A. et al. Identification of distinct gene expression profiles in the synovium of patients with systemic lupus erythematosus. Arthritis Rheum. 56(5), 1579-1588 (2007).

\section{Acknowledgements}

We would like to thank the native English-speaking scientists of Elixigen Company (Huntington Beach, California) for editing our manuscript.

\section{Author contributions}

D.W.L. and G.B.L. designed the experiments. Y.R.H., Z.Q.L. and M.D. contributed to sample collection. L.X.K. and Y.Q.G. performed the bioinformatics and statistical analyses. L.X.K. performed the qRT-PCR experiment. L.X.K. drafted the manuscript. Y.K.L. and B.L.S. helped to revise the manuscript. All authors read and approved the final manuscript.

\section{Funding}

This research was supported by the National Key Research and Development Program of China (2018YFD0501706), and the Guangdong Provincial Promotion Project on Preservation and Utilization of Local Breed of Livestock and Poultry.

\section{Competing interests}

The authors declare no competing interests.

\section{Additional information}

Supplementary information is available for this paper at https://doi.org/10.1038/s41598-020-71206-4.

Correspondence and requests for materials should be addressed to D.L. or Y.L.

Reprints and permissions information is available at www.nature.com/reprints.

Publisher's note Springer Nature remains neutral with regard to jurisdictional claims in published maps and institutional affiliations. 
(c) (i) Open Access This article is licensed under a Creative Commons Attribution 4.0 International cc) Licence, which permits use, sharing, adaptation, distribution and reproduction in any medium or format, as long as you give appropriate credit to the original author(s) and the source, provide a link to the Creative Commons licence, and indicate if changes were made. The images or other third party material in this article are included in the article's Creative Commons licence, unless indicated otherwise in a credit line to the material. If material is not included in the article's Creative Commons licence and your intended use is not permitted by statutory regulation or exceeds the permitted use, you will need to obtain permission directly from the copyright holder. To view a copy of this licence, visit http://creativecommons.org/licenses/by/4.0/.

(C) The Author(s) 2020 